https://doi.org/10.23913/ride.v10i19.587

Artículos Científicos

\title{
Propiedades psicométricas del cuestionario Conflictalk en una muestra de adolescentes mexicanos estudiantes de bachillerato
}

\author{
Psychometric Properties of the Conflictalk Questionnaire in a Sample of \\ Mexican Adolescent High School Students \\ Propriedades psicométricas do questionário Conflictalk em uma amostra de \\ adolescentes mexicanos do ensino médio
}

\author{
Alejandro César Antonio Luna Bernal \\ Universidad de Guadalajara, México \\ aluna642@hotmail.com \\ https://orcid.org/0000-0002-3371-5999
}

\section{Resumen}

El Conflictalk es un cuestionario que evalúa estilos de manejo de conflictos interpersonales (agresivo, pasivo y cooperativo) en población adolescente, el cual ha mostrado ser útil a lo largo de estudios realizados en diversos países. No obstante, el análisis de las propiedades psicométricas del instrumento ha sido poco abordado en la literatura, por lo que se hace necesario contar con mayor información a este respecto, considerando, en particular, el contexto escolar. Por lo anterior, el presente estudio se planteó como objetivo llevar a cabo un análisis de las propiedades psicométricas del Conflictalk a fin de aportar nuevos datos sobre su validez y confiabilidad. La muestra $(N=286)$ estuvo compuesta por estudiantes mexicanos de bachillerato con rango de edad de 15 a 20 años. Además del Conflictalk, los participantes respondieron el Cuestionario sobre estilos de mensajes en el manejo de conflictos (CMMS). Se llevó a cabo un análisis factorial exploratorio, así como uno de confiabilidad, y se estudiaron las diferencias de grupos por edad y género. Además, se analizaron correlaciones entre las escalas de los dos cuestionarios utilizados. En los resultados, se obtuvo una solución trifactorial adecuada al modelo teórico con cargas factoriales de los reactivos superiores a 0.45 , un porcentaje de varianza explicada total de 45.70 , e índices de confiabilidad de los factores de $0.80,0.73$ y 0.82 . 
En el análisis de diferencias de grupo, los varones puntuaron más alto que las mujeres de manera estadísticamente significativa $(p<0.001)$ en el estilo agresivo de manejo de conflictos. Asimismo, en la interacción de edad y género los adolescentes varones del grupo de 17-20 puntuaron más alto en el estilo agresivo, seguidos de los varones de 15 y 16 años, las mujeres de 15 y 16 y, por último, las mujeres de 17 a 20 años; ello, de manera estadísticamente significativa $(p<0.01)$. Este resultado indica una acentuación de las diferencias de género en los grupos de mayor edad. Además de lo anterior, se identificaron correlaciones significativas del Conflictalk con las escalas del CMMS. En conclusión, este estudio aporta información de relevancia para sustentar propiedades aceptables de validez y confiabilidad del Conflictalk en población mexicana adolescente escolarizada de nivel bachillerato. Dentro de las limitaciones y sugerencias, se recomienda para posteriores estudios examinar las relaciones con otros instrumentos y variables de referencia a fin de acumular más elementos de validez convergente y divergente, ampliar el rango de edad de la muestra, así como realizar un nuevo estudio de validación pero desde una perspectiva confirmatoria.

Palabras clave: adolescencia, solución de conflictos, test psicológico.

\section{Abstract}

The Conflictalk questionnaire is an instrument that evaluates interpersonal conflict handling styles (aggressive, passive and cooperative) in adolescent population, which has proved useful throughout studies carried out in different countries. However, the analysis of the psychometric properties of this instrument has been little addressed. For this, it is necessary to have more information about it, considering adolescents' school contexts. In this framework, the aim of this study was to carry out an analysis of the psychometric properties of the Conflictalk in a sample of Mexican high school students in order to provide new data on the validity and reliability of this instrument, considering in particular this type of population. The sample $(N=286)$ was composed of Mexican high school students with an age range of 15-20 years. The participants answered two instruments: the Conflictalk and the Ross-DeWine Conflict Management Message Style Instrument (CMMS). The findings showed a 3-factor solution adequate to the theoretical model with factorial loads above 0.45 , a percentage of total explained variance of 45.70 , and reliability indices of $0.80,0.73$ and 0.82 . In group differences, men scored higher than women, in a statistically significant manner $(p<0.001)$, in the aggressive style of conflict 


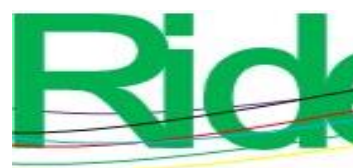

Revista Iberoamericana para la Investigación y el Desarrollo Educativo

ISSN $2007-7467$

management. Likewise, the interaction of gender and age showed differences with statistical significance $(p<0.01)$ in the aggressive style: men of the 17-20 age group scored higher than the men of 15-16 years, followed by the women of 15-16, and finally the women of 17 to 20 years old. This result indicates an accentuation of gender differences in the older age groups. In addition to the above, we also identified significant correlations of the Conflictalk with the CMMS scales. In conclusion, this paper provides relevant information to support acceptable validity and reliability properties of the Conflictalk in the Mexican adolescent at the high school level. Within the limitations and suggestions, it is recommended for further studies to examine the relationships with other instruments and variables in order to accumulate more elements of convergent and divergent validity, extend the age range of the sample, as well as perform a new study of validation from a confirmatory perspective.

Keywords: adolescence, conflict resolution, psychological tests.

\section{Resumo}

O Conflictalk é um questionário que avalia estilos de gerenciamento de conflitos interpessoais (agressivo, passivo e cooperativo) na população adolescente, o que demonstrou ser útil em estudos realizados em vários países. No entanto, a análise das propriedades psicométricas do instrumento tem sido pouco abordada na literatura, sendo necessário ter mais informações a esse respeito, considerando, em particular, o contexto escolar. Portanto, o presente estudo teve como objetivo realizar uma análise das propriedades psicométricas do Conflictalk, a fim de fornecer novos dados sobre sua validade e confiabilidade. A amostra $(\mathrm{N}=286)$ foi composta por estudantes mexicanos do ensino médio, com faixa etária de 15 a 20 anos. Além do Conflictalk, os participantes responderam ao Questionário sobre Estilos de Mensagens de Gerenciamento de Conflitos (CMMS). Foi realizada uma análise fatorial exploratória, bem como uma de confiabilidade, e as diferenças de grupos por idade e sexo foram estudadas. Além disso, foram analisadas correlações entre as escalas dos dois questionários utilizados. Nos resultados, uma solução de três fatores adequada ao modelo teórico foi obtida com cargas fatoriais de reagentes maiores que 0,45, porcentagem da variância total explicada de 45,70 e índices de confiabilidade de fatores de 0,80, 0,73 e 0,82. Na análise das diferenças entre os grupos, os homens pontuaram mais alto que as mulheres de forma estatisticamente significante $(p<0,001)$ no estilo agressivo de gerenciamento de conflitos. Da mesma forma, na interação idade e sexo, os adolescentes do 


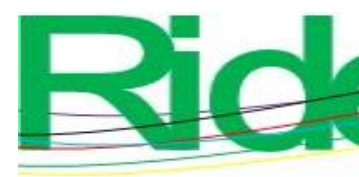

Revista Iberoamericana para la Investigación y el Desarrollo Educativo

ISSN $2007-7467$

grupo 17-20 tiveram uma pontuação mais alta no estilo agressivo, seguidos pelos homens de 15 e 16 anos, as mulheres de 15 e 16 anos e, finalmente, as mulheres de 17 a 20 anos; isso, de forma estatisticamente significante ( $\mathrm{p}<0,01)$. Este resultado indica uma acentuação das diferenças de gênero nas faixas etárias mais velhas. Além do acima, foram identificadas correlações significativas do Conflictalk com as escalas do CMMS. Em conclusão, este estudo fornece informações relevantes para apoiar propriedades aceitáveis de validade e confiabilidade do Conflictalk em adolescentes mexicanos de nível médio. Dentro das limitações e sugestões, recomenda-se que estudos subsequentes examinem as relações com outros instrumentos e variáveis de referência, a fim de acumular mais elementos de validade convergente e divergente, ampliar a faixa etária da amostra e realizar um novo estudo de validação, mas de uma perspectiva confirmatória.

Palavras-chave: adolescência, resolução de conflitos, teste psicológico.

Fecha Recepción: Julio 2019

Fecha Aceptación: Diciembre 2019

\section{Introducción}

La expresión estilo de manejo de conflictos hace referencia a "una cierta manera de afrontar los conflictos la cual un individuo tiende a asumir habitualmente en su vida cotidiana" (Luna, Valencia, Nava y Ureña, 2019, p. 158). Esta definición coincide con la de otros autores que han considerado a dichos estilos como una disposición o inclinación de cada persona a tratar los conflictos interpersonales de un modo característico (Blake y Mouton, 1970; Filley, 1985; Laca, 2005). Por ejemplo, una persona puede estar inclinada a imponer su posición a las contrapartes con las que se encuentra en sus diversos conflictos, tendiendo a afirmar su interés propio (estilo dominante). En cambio, otra persona tal vez tenga la inclinación a ceder ante las pretensiones de los demás, prefiriendo satisfacer las demandas e intereses de los otros, incluso a expensas de los suyos (estilo complaciente). Una tercera persona tal vez se incline a manejar los conflictos de modo tal que ambas partes obtengan plenamente la satisfacción de sus respectivos intereses (estilo colaborativo). Cabe señalar que en la literatura del área existen varios modelos entre los cuales hay divergencias en cuanto al número y características de estos estilos (Luna, 2018; Rahim, 2001). 


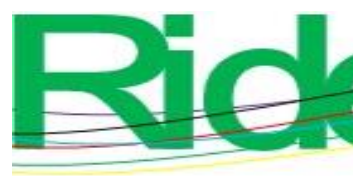

Revista lberoamericana para la Investigación y el Desarrollo Educativo

ISSN $2007-7467$

En la actualidad, el estudio de los estilos de manejo de conflictos que los adolescentes emplean en los diversos contextos relacionales en los que se desenvuelven, y especialmente en el ámbito de las relaciones con sus pares en el contexto escolar, ha recibido una creciente atención por parte de los investigadores (Luna, Mejía y Laca, 2017). Este creciente interés puede explicarse por varios factores. Por una parte, en la literatura contemporánea es perceptible un reconocimiento cada vez mayor de que los conflictos son inherentes a las relaciones humanas y pueden ser transformados constructivamente si se abordan de una manera adecuada (Paris, 2009; Ramón, García y Olalde, 2019). Por otra parte, la formación de competencias para el manejo constructivo de conflictos se ha considerado un elemento en la educación de los adolescentes y jóvenes que favorece mejores formas de convivencia, contribuye a la disminución de la violencia y fortalece una cultura de paz (De la Rosa, Ángeles y Pérez, 2018; Pegalajar, 2018). De acuerdo con Azzolini (2017), "la violencia en las escuelas es uno de los grandes obstáculos para que los niños gocen efectivamente del derecho a la educación" (p. 56). Por ello, "se deben impulsar planes de estudios en los que se incorporen la equidad, la no violencia y la resolución pacífica de conflictos entre los principales objetivos educativos" (Santamaría-Cárdaba, 2019, p. 72).

En ese marco, la necesidad de contar con mayores conocimientos acerca de cómo los adolescentes afrontan los conflictos que se les presentan en el curso de su vida cotidiana en el contexto escolar se ha convertido en un aspecto de relevancia tanto para entender las dinámicas interpersonales que les afectan como para generar propuestas de formación, atención e intervención.

Junto con la necesidad de contar con mayores conocimientos surge la de tener instrumentos válidos y confiables que permitan una evaluación con las garantías psicométricas adecuadas. En la literatura internacional existen diversos modelos e instrumentos para describir y evaluar estilos de manejo de conflictos en diversos contextos; entre los más conocidos es posible mencionar el Thomas-Kilmann Conflict Management of Differences (MODE) de Thomas y Kilmann (1974), el Rahim's Organizational Conflict Inventory-II (ROCI-II) de Rahim (1983) y el Conflict Management Message Style Instrument (CMMS) de Ross y DeWine (1988). Aunque estos instrumentos han sido empleados en diversos estudios para evaluar estilos de manejo de conflictos en población adolescente — por ejemplo: Chang y Zelihic (2013), De 
Conti (2014) y Luna y Laca (2014) — lo cierto es que no han sido diseñados específicamente para este grupo etario.

El Conflictalk es un instrumento propuesto por Kimsey y Fuller (2003) para evaluar estilos de manejo de conflictos específicamente en población adolescente. El enfoque del instrumento es comunicativo, ya que considera dichos estilos a partir del tipo de mensajes verbales que los individuos tienden a utilizar en sus interacciones conflictivas. Aunque ya existía el cuestionario CMMS de Ross y DeWine (1988) para evaluar estilos de mensajes verbales en situaciones de conflicto, Kimsey y Fuller (2003) consideraron que era necesario un nuevo instrumento que estuviera adaptado al lenguaje específico de los adolescentes. En efecto, de acuerdo con estos autores, el lenguaje de los adolescentes es parte de su cultura y es considerado por ellos como el vehículo de la comunicación más auténtica; así, serán más hábiles para manejar sus conflictos en la medida en que puedan utilizar su lenguaje característico para expresarse en dichas situaciones. De ahí la importancia de evaluar los estilos de manejo de conflictos a partir de los estilos de mensajes expresados en el propio lenguaje de los adolescentes, según los autores mencionados.

Kimsey y Fuller (2003) desarrollaron el Conflictalk trabajando con una muestra de 500 estudiantes de educación básica (elementary school, $4 .^{\circ}$ y $5 .^{\circ}$ grado; $n=222$ ), media básica (middle school, del 6. ${ }^{\circ}$ al 8. ${ }^{\circ}$ grado; $n=133$ ) y bachillerato (high school, del 9. ${ }^{\circ}$ al 12. ${ }^{\circ}$ grado; $n$ =145). En su versión original, el instrumento quedó compuesto por 18 reactivos divididos en tres escalas: a) el estilo enfocado hacia sí mismo (self-focus) (rinoceronte-agresivo), $b$ ) el estilo enfocado hacia el problema (problem focus) (delfín-cooperativo) y c) el estilo enfocado hacia los otros (other-focus) (avestruz-pasivo). Los símbolos de animales fueron utilizados por los autores para facilitar la comprensión de cada uno de estos estilos.

De acuerdo con Kimsey y Fuller (2003), el estilo enfocado hacia sí mismo (rinoceronteagresivo) consiste, como su propio nombre lo indica, en estar centrado en sí mismo, queriendo que las cosas se hagan a la manera propia, actuando de manera agresiva y autoritaria frente al conflicto. Se trata de un estilo dominante en que el adolescente intenta satisfacer sus propios intereses incluso a expensas de los demás. El estilo enfocado hacia el problema (delfíncooperativo) consiste en mostrar interés por la causa del conflicto y en identificar concretamente el problema en colaboración con el otro. Se trata de un estilo colaborativo donde el interés se centra en encontrar la mejor solución al problema, así como en actuar cooperativamente. Por 
último, el estilo enfocado hacia los otros (avestruz-pasivo) se basa en la creencia de que todo conflicto es siempre algo malo que debe evitarse, así como en el deseo de que la otra parte sea feliz. Se trata de un estilo en el que el adolescente actúa de manera pasiva y evitativa frente al conflicto (Kimsey y Fuller, 2003; Garaigordobil, Machimbarrena y Maganto 2016).

El Conflictalk ha demostrado ser de utilidad en estudios con población adolescente realizados en diversos países como Estados Unidos — por ejemplo, Donegani y Séguin (2018) y Lane, Ybarra, Zajac y Vierra (2005)—, Israel (Hochhauser, Weiss y Gal, 2018), España por ejemplo, Garaigordobil (2009, 2012, 2017), Garaigordobil y Maganto (2011), Garaigordobil y Martínez (2015), Garaigordobil et al. (2016) y Garaigordobil, Maganto, Pérez y Sansinenea (2009) - y México — por ejemplo: De la Rosa et al. (2018), Laca, Alzate, Sánchez, Verdugo y Guzmán (2006) y Luna y Laca (2017). Es por ello por lo que, actualmente, se ha convertido en una herramienta de referencia para esta área de investigación.

La traducción del Conflictalk a la lengua castellana fue llevada a cabo en México por Laca et al. (2006) en un estudio con una muestra de 526 niños y adolescentes de la ciudad de Colima, Colima, los cuales tenían un rango de edad de 9 a 17 años. En España, Garaigordobil (2009) reportó estudios de validación del Conflictalk realizados con muestras $(N=313,285 \mathrm{y}$ 123) de adolescentes de 15 a 17 años en los que se obtuvieron datos adecuados de confiabilidad y validez. Más recientemente, Garaigordobil et al. (2016) presentaron una adaptación española del Conflictalk en un estudio con una muestra de 2283 participantes del País Vasco, con un rango de edad de 12 a 17 años.

Considerando los mencionados antecedentes, así como la importancia y el actual interés que existe por estudiar los procesos a través de los cuales los estudiantes adolescentes manejan los conflictos interpersonales que se les presentan con sus pares en el contexto escolar, es que el presente estudio se planteó llevar a cabo un análisis de las propiedades psicométricas del Conflictalk en una muestra de adolescentes mexicanos estudiantes de bachillerato, a fin de aportar nuevos datos sobre la validez y confiabilidad de este instrumento, considerando en particular este tipo de población. Lo anterior debido fundamentalmente a las razones que se exponen a continuación.

En primer lugar, en los estudios de validación llevados a cabo en España (Garaigordobil, 2009; Garaigordobil et al., 2016) los autores aportaron abundantes elementos de validez convergente y discriminante, pero no presentaron un análisis de la estructura factorial del 
Conflictalk. Por su parte, en el estudio de validación llevado a cabo en México por Laca et al. (2006), si bien se llevó a cabo un análisis factorial, la distribución de los reactivos entre las escalas varió sensiblemente con respecto al instrumento original.

Aunado a lo anterior, es importante notar que en todos estos estudios de validación de la versión castellana del Conflictalk (Garaigordobil, 2009; Garaigordobil et al., 2016; Laca et al., 2006) se consideraron rangos de edad de los participantes hasta los 17 años; sin embargo, en la actualidad, el rango de los 17 a los 20 años suele considerarse como correspondiente al periodo de la adolescencia tardía (Ibarra y Jacobo, 2014; Iglesias, 2013; Steinberg, 2017). Un grupo de estudio de la Organización Mundial de la Salud [OMS] (1986), por ejemplo, consideró la siguiente división: adolescencia inicial de 10 a 14 años, adolescencia media de 14 a 17 años y adolescencia final de 17 a 20 años.

Relacionado con esto último, conviene mencionar que en el estudio original de Kimsey y Fuller (2003) se realizó un análisis factorial exploratorio de los reactivos en cada una de las tres submuestras de adolescentes. Y resultó que los tres factores se agruparon como se tenía hipotetizado en las submuestras de participantes de educación básica (elementary) y media básica (middle), pero en la submuestra de adolescentes de bachillerato (high school) varió ligeramente, ya que hubo un par de reactivos (el uno y el dos) que no presentaron cargas factoriales adecuadas (en ambos casos fueron menores a 0.40), y el reactivo 16 presentó una asignación ambigua entre el estilo enfocado en la otra parte y el enfocado en sí mismo (cargas factoriales de 0.40 y 0.43 , respectivamente). Según dichos autores, este hallazgo podría sugerir "que a medida que maduran los sujetos hay una evolución en su estilo de mensajes en el manejo de conflictos" (Kimsey y Fuller, 2003, p. 76).

Debido a todo lo anterior, se consideró oportuno realizar el presente estudio que pretende evaluar las propiedades psicométricas del Conflictalk en una muestra de adolescentes mexicanos estudiantes de bachillerato, de 15 a 20 años. Para ello se analizará la estructura factorial de dicho instrumento, así como su consistencia interna, y las posibles diferencias por edad y género.

Por otra parte, es importante considerar que hasta el día de hoy la mayoría de los estudios realizados en México sobre estilos de manejo de conflictos en adolescentes — por ejemplo, Luna y Laca (2014), Luna (2014, 2017) y Luna et al. (2017) — han estado basados en el modelo de Ross y DeWine (1988). Debido a ello, se consideró pertinente en el presente trabajo, además de lo señalado anteriormente, el analizar también las relaciones que pudieran darse entre los estilos 


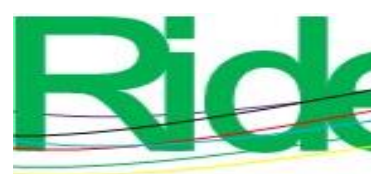

Revista Iberoamericana para la

Investigación y el Desarrollo Educativo

ISSN $2007-7467$

de manejo de conflictos del modelo de Kimsey y Fuller (2003) y los estilos del modelo de Ross y DeWine (1988), ya que ello contribuirá a las discusiones que comparen los resultados de los estudios realizados con uno y otro instrumento, además de que agregará un criterio de validez convergente al presente análisis.

Ross y DeWine (1988) propusieron tres estilos de manejo de conflictos: a) el estilo centrado en uno mismo (concern for self), el cual se presenta cuando en el manejo de un conflicto el individuo se enfoca en su propio interés o postura personal, sin considerar el interés de la otra persona; $b$ ) el estilo centrado en la otra parte (concern for other): cuando el sujeto se enfoca en satisfacer el interés de la contraparte, incluso dejando de lado los intereses propios, y c) el estilo centrado en el problema (concern for issue), el cual se manifiesta cuando el individuo decide enfocarse en el asunto que es objeto de conflicto, expresando mensajes en los que invita a la otra parte a colaborar o a convenir en alguna solución que satisfaga al menos parcialmente los intereses de ambas. Para evaluar dichos estilos, Ross y DeWine propusieron el ya mencionado CMMS, cuyas características serán señaladas en el apartado siguiente.

Sintetizando todo lo anterior, el presente estudio se ha planteado cuatro objetivos específicos: 1) analizar la estructura factorial del Conflictalk en una muestra de adolescentes estudiantes de bachillerato con edades de 15 a 20 años; 2) analizar la consistencia interna del mencionado cuestionario con los datos de la muestra de estudio; 3) analizar diferencias de edad y género en los puntajes de las escalas que componen el Conflictalk, y 4) analizar las correlaciones entre las mencionadas tres escalas del Conflictalk y las correspondientes del cuestionario CMMS de Ross y DeWine (1988).

En relación con este último objetivo, se espera como hipótesis el que se presenten correlaciones positivas estadísticamente significativas: $a$ ) entre el estilo centrado en uno mismo del CMMS y el enfocado hacia sí mismo (rinoceronte-agresivo) del Conflictalk, $b$ ) entre el estilo centrado en la otra parte del CMMS y el enfocado hacia los otros (avestruz-pasivo) y $c$ ) entre el estilo centrado en el problema del CMMS y el enfocado hacia el problema (delfín-cooperativo) del Conflictalk. Lo anterior debido a la evidente afinidad teórica entre cada uno de estos estilos derivada del hecho de que Kimsey y Fuller (2003), según su propia afirmación, tomaron al CMMS como un "prototipo" (prototype) en el diseño de su cuestionario. 


\section{Método}

\section{Participantes}

El estudio se llevó a cabo en una escuela preparatoria pública ubicada dentro de la Zona Metropolitana de Guadalajara, Jalisco (México). La población total de la escuela participante fue de 1350 estudiantes, de los cuales 635 eran mujeres (47.0\%) y 715 hombres (53.0 \%). El muestreo se llevó a cabo eligiendo al azar un grupo de cada grado (semestre). Así, la muestra estuvo compuesta por 286 alumnos. De estos, 52.1\% fueron mujeres y $47.9 \%$ hombres. El rango de edad fue de 15 a 20 años, con media aritmética $(M)$ de 16.52 años y desviación estándar (DE) de 1.05. Para los fines del presente estudio se formaron dos grupos de edad: 15 y 16 años (57.3\%) y 17 a 20 años (42.7\%). Esta división corresponde, en términos generales, a los periodos de la adolescencia media y tardía, respectivamente (Ibarra y Jacobo, 2014; Iglesias, 2013; OMS, 1986; Steinberg, 2017). En la tabla 1 puede observarse la distribución de los participantes por edad, en función del género.

Tabla 1. Distribución de los participantes según género y edad

\begin{tabular}{|l|c|c|c|}
\hline & Mujeres & Hombres & Total \\
\hline 15 a 16 años & $89(31.1 \%)$ & $75(26.2 \%)$ & $164(57.3 \%)$ \\
\hline 17 a 20 años & $60(21.0 \%)$ & $62(21.7 \%)$ & $122(42.7 \%)$ \\
\hline Total & $149(52.1 \%)$ & $137(47.9 \%)$ & $286(100 \%)$ \\
\hline
\end{tabular}

Nota: Los porcentajes son con respecto a la muestra total.

Fuente: Elaboración propia

\section{Instrumentos}

\section{Instrumento para evaluar los estilos de mensajes en el manejo de conflictos en jóvenes y adolescentes (Conflictalk questionnaire)}

Este cuestionario informa sobre la frecuencia con que los participantes perciben utilizar los tres estilos de manejo de conflictos propuestos por Kimsey y Fuller (2003). Como ya se mencionó, en su versión original está compuesto por 18 reactivos divididos en tres escalas: $a$ ) estilo enfocado hacia sí mismo (rinoceronte-agresivo), $b$ ) estilo enfocado hacia el problema (delfín-cooperativo) y c) estilo enfocado hacia los otros (avestruz-pasivo). 


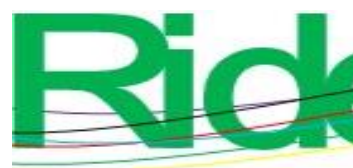

Revista lberoamericana para la Investigación y el Desarrollo Educativo

ISSN $2007-7467$

Cada uno de los reactivos es una oración que representa un mensaje dado por un individuo a su contraparte en una situación de conflicto interpersonal, por ejemplo: "No se me da bien esto. Simplemente, no sé cómo hacer que te sientas mejor” (reactivo dos) o "¿Qué está pasando? Necesitamos hablar” (reactivo tres) o “¡Cállate! ¡No tienes razón! No voy a escucharte" (reactivo ocho). Para contestar, se le pide al participante que indique con qué frecuencia él ha utilizado, en sus conflictos, mensajes similares a los representados en cada reactivo. El formato de respuesta es una escala Likert de cinco puntos: $1=$ Nunca, 2 = Rara vez, 3 = Algunas veces, $4=$ Frecuentemente y $5=$ Siempre. Para la calificación del cuestionario se obtienen las medias y desviaciones estándar de los participantes en cada una de las tres escalas.

Como se señaló, el Conflictalk fue desarrollado por Kimsey y Fuller (2003). Los autores reportaron una confiabilidad alfa de Cronbach de $0.81,0.87$ y 0.63 para las escalas enfocado hacia sí mismo (rinoceronte-agresivo), enfocado hacia el problema (delfín-cooperativo) y enfocado hacia los otros (avestruz-pasivo), respectivamente. Los índices de confiabilidad reportados por Garaigordobil et al. (2016) para la versión castellana de estas mismas escalas fueron de $0.77,0.90$ y 0.70 respectivamente.

\section{Cuestionario sobre estilos de mensajes en el manejo de conflictos (CMMS)}

Se trata de un cuestionario de autoinforme que evalúa los estilos de manejo de conflictos con base en el tipo de mensajes verbales que los individuos perciben utilizar durante las situaciones de interacción conflictiva. Se compone de tres escalas: estilo enfocado en uno mismo, estilo enfocado en la otra parte y estilo enfocado en el problema.

De manera similar al Conflictalk, cada uno de los reactivos del CMMS es una oración que representa un mensaje verbal dado por un individuo a su contraparte en una situación de conflicto interpersonal, por ejemplo: “Cómo puedo hacerte sentir bien otra vez?” (reactivo dos) o "Estoy muy molesto por algunas cosas que están pasando; ¿podemos hablar sobre ellas?" (reactivo tres), o bien “¡Cállate, estás equivocado(a)! No quiero oír nada más de lo que tengas que decir" (reactivo ocho). Para contestar, se le pide al participante que indique con qué frecuencia él ha utilizado mensajes similares a los representados por cada reactivo. El formato de respuesta es una escala Likert de cinco puntos: $1=$ Nunca, $2=$ Rara vez, $3=$ Algunas veces, 4 = Frecuentemente y 5 = Siempre. Para la calificación del CMMS se obtienen las medias y desviaciones estándar de los participantes en cada una de las tres escalas. 


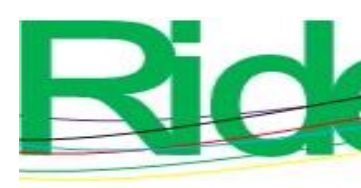

Revista lberoamericana para la Investigación y el Desarrollo Educativo

ISSN $2007-7467$

Ross y DeWine (1988) diseñaron y validaron originalmente el CMMS tras una serie de estudios efectuados con estudiantes universitarios en Estados Unidos. Mejía y Laca (2006) y Laca, Mejía y Mayoral (2011) hicieron la traducción y validación a la lengua castellana, en estudios con muestras de jóvenes universitarios de Colima y del País Vasco, respectivamente. Más recientemente, Luna y Laca (2014) hicieron un estudio de validación de la traducción castellana en un estudio en el que participaron 1074 estudiantes mexicanos de secundaria, bachillerato y licenciatura, con edades de 11 a 25 años. Estos autores realizaron análisis factoriales exploratorios y confirmatorios del CMMS. Como resultado, el instrumento quedó compuesto por 13 reactivos divididos en tres escalas: $a$ ) estilo enfocado en uno mismo, $b$ ) estilo enfocado en la otra parte y $c$ ) estilo enfocado en el problema, con índices de confiabilidad alfa de Cronbach de $0.68,0.72$ y 0.83 , respectivamente.

\section{Procedimiento}

El presente estudio se apegó en todo su desarrollo a los principios éticos y código de conducta de la American Psychological Association [APA] (2017), así como a los aspectos relativos del Código Ético del Psicólogo de la Sociedad Mexicana de Psicología (2010).

La aplicación de los instrumentos se llevó a cabo dentro del aula de clases, una vez que se obtuvieron los permisos correspondientes por parte de las autoridades del plantel. El investigador, acompañado de dos asistentes previamente capacitados, ingresó a cada aula, informó a los estudiantes acerca de los objetivos de la investigación y se les hizo una invitación a participar de una manera completamente voluntaria y anónima; se les permitió salir del aula a quienes no desearan participar, así como a quienes decidieran no continuar una vez comenzada la aplicación. No se les solicitó información que pudiera identificarles de manera individual, solo algunos datos generales para efectos estadísticos (escuela, grado, género y edad). Se les garantizó que la información sería tratada de manera estrictamente confidencial y que los datos serían utilizados para fines exclusivamente científicos. Se les informó también que en este tipo de instrumentos no hay respuestas buenas o malas, sino que lo importante para la investigación es que sus respuestas sean lo más sinceras posibles. 


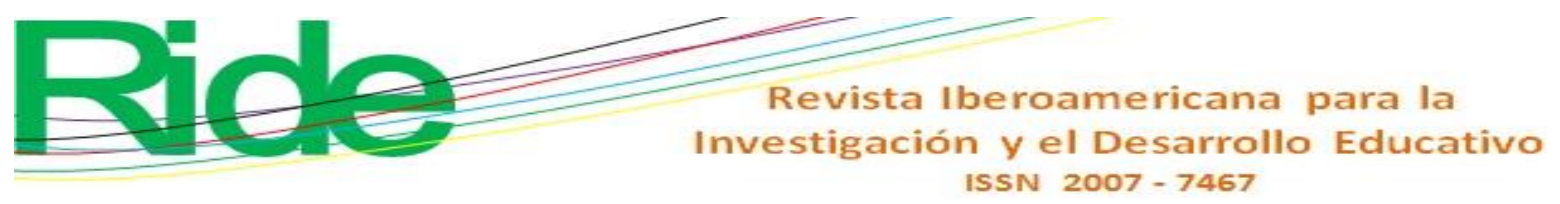

\section{Análisis estadístico}

Con el fin de analizar la estructura factorial del Conflictalk se llevó a cabo un análisis factorial exploratorio. La pertinencia de la técnica de este análisis para los datos muestrales se determinó a través de dos procedimientos: a) el examen de la medida de adecuación muestral de Kaiser-Meyer-Olkin y b) la prueba de esfericidad de Bartlett. Enseguida se procedió a la extracción de factores empleando el método de ejes principales. Este método es aconsejable para examinar la estructura subyacente a los reactivos de una escala, en situaciones en las que se emplean escalas Likert de al menos cinco opciones y se tienen distribuciones no muy alejadas de la normalidad (Lloret, Ferreira, Hernández y Tomás, 2014). Como método de rotación se eligió una de tipo ortogonal (Varimax), ya que fue la empleada por Kimsey y Fuller (2003) en el estudio de validación del instrumento original. Para la elección del número de componentes a retener, se consideraron los siguientes criterios (Moral de la Rubia, 2016): a) que el componente tuviera un autovalor mayor que uno, $b$ ) que el componente se situara por encima del punto de inflexión de la curva en el gráfico de sedimentación, c) que cada componente estuviera compuesto con más de dos variables con saturaciones mayores a $0.40, d$ ) que fuera posible ubicar a cada variable en un solo componente con base en la saturación más alta de dicha variable, siempre que sea mayor a 0.40 y e) que el componente fuera identificable e interpretable, con base en el constructo y en el diseño del instrumento.

Hecho lo anterior, se calculó el coeficiente de confiabilidad alfa de Cronbach para cada factor resultante, así como las puntuaciones medias y desviaciones estándar correspondientes.

Con el fin de identificar posibles efectos de edad y género en las variables de estudio, se llevó a cabo un análisis multivariado de la varianza (Manova) con diseño de 2 x 2 (dos niveles de género por dos de edad).

Para identificar posibles correlaciones significativas entre las escalas del Conflictalk y del CMMS, se llevó a cabo un análisis de correlaciones parciales introduciendo la edad y el género como variables de control.

Todos los cálculos se realizaron empleando el programa estadístico SPSS 21 (IBM Corporation, 2012). 


\section{Resultados}

El análisis factorial exploratorio se llevó a cabo en tres rondas. Inicialmente, el método de extracción arrojó una solución de cuatro factores; sin embargo, el Factor IV quedó compuesto únicamente con dos reactivos (dos y cuatro). Por tanto, se decidió prescindir de dichos reactivos, de conformidad a los criterios establecidos. En una segunda ronda de análisis, llevada a cabo con 16 reactivos, el método de extracción arrojó una solución trifactorial; no obstante, el reactivo 18 mostró una asignación ambigua ya que cargó con 0.534 en el segundo factor y con 0.431 en el tercero. Finalmente, una tercera ronda de análisis llevada a cabo con 15 reactivos dio por resultado una solución que cumplió con todos los criterios establecidos. Se reportan a continuación los valores de esta solución final.

Se obtuvieron valores adecuados en la medida Kaiser-Meyer-Olkin de adecuación muestral y en la prueba de esfericidad de Bartlett $\left(K M O=0.80 ; J i^{2}{ }_{(105)}=1424.77, p<0.001\right)$, lo que indica la pertinencia del análisis factorial exploratorio. El método de extracción arrojó una solución de tres factores que explica en total $45.70 \%$ de la varianza con un índice alfa de Cronbach de 0.77 para el total de la escala. En la tabla 2 se muestran los pesos factoriales, el autovalor, la varianza explicada y los índices de confiabilidad obtenidos para cada factor en esta solución final. 
Tabla 2. Análisis factorial exploratorio del Conflictalk $(N=286)$

\begin{tabular}{|c|c|c|c|}
\hline \multirow[t]{2}{*}{ Reactivos } & \multicolumn{3}{|c|}{ Factor } \\
\hline & I & II & III \\
\hline 11. Funcionará si trabajamos juntos. & 0.755 & 0.052 & -0.128 \\
\hline 12. Trabajaremos para sacar esto adelante. & 0.749 & -0.111 & -0.091 \\
\hline 17. Necesitamos concretar juntos cuál es el problema. & 0.679 & -0.014 & 0.159 \\
\hline 7. Vamos a hablar sobre esto y a encontrar una solución. & 0.654 & -0.060 & 0.133 \\
\hline 5. Tenemos que concretar eso. & 0.582 & -0.005 & 0.090 \\
\hline 3. ¿Qué está pasando? Necesitamos hablar. & 0.544 & -0.108 & 0.060 \\
\hline 6. Quisiera que pudiéramos evitar todo este asunto. & 0.453 & 0.040 & 0.256 \\
\hline 9. ¡Es culpa tuya! Y no voy a ayudarte. & -0.037 & 0.778 & 0.136 \\
\hline 8. ¡Cállate! ¡No tienes razón! No voy a escucharte. & -0.075 & 0.748 & 0.249 \\
\hline 10. Harás lo que yo te digo. ¡Te voy a obligar! & -0.093 & 0.687 & 0.071 \\
\hline 1. ¿No te das cuenta de lo estúpido/a que eres? & 0.023 & 0.555 & 0.257 \\
\hline $\begin{array}{l}\text { 15. Esto no va a ninguna parte, olvidemos todo el asunto, } \\
\text { ¿de acuerdo? }\end{array}$ & 0.190 & 0.088 & 0.691 \\
\hline 13. De acuerdo, me rindo, lo que tú quieras. & 0.063 & 0.112 & 0.587 \\
\hline $\begin{array}{l}\text { 16. Si no quieres hacerlo, olvídalo; ya se lo pediré a } \\
\text { algún otro. }\end{array}$ & -0.031 & 0.313 & 0.582 \\
\hline $\begin{array}{l}\text { 14. No quiero hacer esto nunca más. Vamos cada uno } \\
\text { por su lado. }\end{array}$ & 0.077 & 0.218 & 0.576 \\
\hline Autovalor & 1.79 & 2.92 & 2.14 \\
\hline Porcentaje de varianza explicada & 11.93 & 19.49 & 14.29 \\
\hline Confiabilidad alfa de Cronbach & 0.823 & 0.802 & 0.733 \\
\hline
\end{tabular}

Nota. Técnica de extracción: Factorización de ejes principales. Rotación: Varimax.

Fuente: Elaboración propia

Como puede observarse, los reactivos que comprenden el Factor I corresponden al estilo enfocado en el problema (delfín-cooperativo) del cuestionario original; el Factor II corresponde al estilo enfocado en sí mismo (rinoceronte-agresivo) y el Factor III al estilo enfocado en la otra parte (avestruz-evitativo). Las puntuaciones medias obtenidas por los participantes en cada una 
de las escalas mencionada fueron de $3.22(\mathrm{DE}=0.89), 1.70(\mathrm{DE}=0.83)$ y $2.26(\mathrm{DE}=0.86)$, respectivamente.

Una vez realizado el Manova, no resultó ser estadísticamente significativo el efecto principal de la variable de edad (lambda de Wilks $(3 / 280)=0.987, p=0.308)$; pero sí el de género (lambda de Wilks $(3 / 280)=0.915, p<0.001)$ y el de interacción de género y edad (lambda de Wilks $(3 / 280)=0.954, p<0.01)$. En ambos casos, dicha diferencia estadísticamente significativa correspondió al estilo enfocado en sí mismo (rinoceronte-agresivo), siendo $F_{(1 / 285)}=24.021$ ( $p$ $<0.001)$ en el primer caso, y $F_{(1 / 285)}=8.098(p<0.01)$ en el segundo. En la tabla 3 se presentan las puntuaciones medias y desviaciones estándar correspondientes. Como se puede observar, en el estilo agresivo, los hombres de 17 a 20 años obtienen las puntuaciones más altas, seguidos de los varones de 15 y 16 años. El grupo con la puntuación más baja en dicho estilo fue el de las mujeres con edades de 17 a 20 años.

Tabla 3. Medias y desviaciones estándar en el estilo agresivo por género y edad $(N=286)$

\begin{tabular}{|l|c|c|c|}
\hline & $\begin{array}{c}\text { Mujeres } \\
M(\mathrm{DE})\end{array}$ & $\begin{array}{c}\text { Hombres } \\
M(\mathrm{DE})\end{array}$ & $\begin{array}{c}\text { Total } \\
\end{array}$ \\
\hline 15 a 16 años & $1.57(0.78)$ & $1.73(0.75)$ & $1.64(0.77)$ \\
\hline 17 a 20 años & $1.40(0.61)$ & $2.13(0.99)$ & $1.77(0.90)$ \\
\hline Total & $1.50(0.72)$ & $1.91(0.89)$ & $1.70(0.83)$ \\
\hline
\end{tabular}

Fuente: Elaboración propia

Finalmente, con el objetivo de explorar las relaciones entre los estilos de manejo de conflictos del Conflictalk y los del cuestionario CMMS de Ross y DeWine (1988), se llevó a cabo un análisis de correlación de Pearson entre las escalas de ambos instrumentos. Debido a que, como se señaló, se encontró un efecto del género y de su interacción con la edad, se decidió emplear el análisis de correlación parcial a fin de controlar dichas variables. Los resultados se muestran en la tabla 4. Como se puede observar, tanto el estilo agresivo como el evitativo del Conflictalk correlacionaron positivamente de manera estadísticamente significativa con el estilo enfocado en sí mismo del cuestionario CMMS. Por su parte, el estilo cooperativo del Conflictalk correlacionó significativamente con los estilos enfocado en la otra parte y enfocado en el problema del CMMS. 
Tabla 4. Coeficientes de correlación parcial entre estilos de manejo de conflictos, controlando género $(N=282)$

\begin{tabular}{|l|c|c|c|}
\hline & & $\begin{array}{c}\text { Cuestionario } \\
\text { CMMS }\end{array}$ & \\
\hline Conflictalk & $\begin{array}{c}\text { Estilo enfocado en } \\
\text { sí mismo }\end{array}$ & $\begin{array}{c}\text { Estilo enfocado en } \\
\text { la otra parte }\end{array}$ & $\begin{array}{c}\text { Estilo enfocado en } \\
\text { el problema }\end{array}$ \\
\hline $\begin{array}{l}\text { Estilo enfocado en sí mismo } \\
\text { (rinoceronte-agresivo) }\end{array}$ & $0.447^{* * *}$ & 0.002 & -0.073 \\
\hline $\begin{array}{l}\text { Estilo enfocado en la otra parte } \\
\text { (avestruz-evitativo) }\end{array}$ & $0.287^{* * *}$ & 0.061 & 0.067 \\
\hline $\begin{array}{l}\text { Estilo enfocado en el problema } \\
\text { (delfín-cooperativo) }\end{array}$ & 0.038 & $0.495^{* * *}$ & $0.621 * * *$ \\
\hline
\end{tabular}

Nota: $* * * p<0.001$.

Fuente: Elaboración propia

\section{Discusión}

Como se señaló, el presente trabajo se planteó como objetivos específicos el analizar: $a$ ) la estructura factorial del Conflictalk en una muestra de adolescentes estudiantes de bachillerato con edades de 15 a 20 años; b) la consistencia interna del mencionado cuestionario; $c$ ) las posibles diferencias por edad y género, y $d$ ) las correlaciones entre las escalas del Conflictalk y las del cuestionario CMMS.

Con respecto al primer objetivo, los resultados sugieren una solución trifactorial, con buenos indicadores de validez de acuerdo con los criterios considerados (Moral de la Rubia, 2016). Dicha solución es congruente, en términos generales, con el modelo original de Kimsey y Fuller (2003), misma que ha sido tomada como referencia en la mayor parte de los estudios llevados a cabo con este instrumento a nivel internacional, a los cuales se hizo referencia más arriba. 


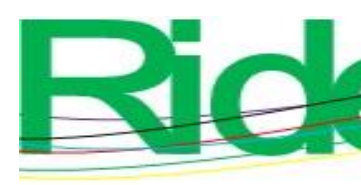

Revista Iberoamericana para la Investigación y el Desarrollo Educativo

ISSN $2007-7467$

En el marco de lo anterior, conviene destacar el hecho de que en la escala del estilo enfocado en la otra parte (avestruz-evitativo) los reactivos dos y cuatro fueron eliminados durante el análisis en el presente estudio, mientras que se incorporó a ella el reactivo 16, que originalmente pertenecía a la escala enfocado en sí mismo (rinoceronte-agresivo). Por un lado, este resultado es congruente con el estudio original de Kimsey y Fuller (2003), donde, en la submuestra de adolescentes de bachillerato (high school), el reactivo dos tampoco obtuvo una carga factorial adecuada y el reactivo 16 presentó una asignación ambigua entre las escalas enfocado en sí mismo y enfocado en la otra parte. Por otro lado, al revisar el contenido de los cuatro reactivos que cargaron en esta escala en el presente estudio (ver tabla 2), es posible observar una congruencia entre ellos en el sentido de que todos contienen mensajes orientados hacia terminar o finalizar una discusión, lo cual es consistente con el carácter evitativo de este estilo de manejo de conflictos.

Por su parte, con respecto al segundo objetivo, los índices de confiabilidad alfa de Cronbach resultaron aceptables en un caso y buenos en los otros dos, ya que el primero fue superior a 0.70 y los otros a 0.80 (George y Mallery, 2003). Este resultado es similar, en términos generales, al obtenido en el estudio previo llevado a cabo por Garaigordobil et al. (2016) en el País Vasco al analizar la confiabilidad de la versión castellana del Conflictalk.

Pasando al tercer objetivo, en relación con las diferencias por género, como se pudo observar, los hombres presentaron en general una inclinación hacia puntuaciones más altas que las mujeres en el estilo enfocado en sí mismo (rinoceronte-agresivo). Este resultado es congruente con la literatura del área en la que se ha encontrado de manera consistente una mayor inclinación de los varones hacia las formas agresivas de manejo de conflictos (Garaigordobil et al., 2016; Luna, De Gante y Gómez, 2018). La explicación más aceptada sugiere que los procesos de socialización diferencial de género, basados en los roles y estereotipos de género tradicionales, favorecen en los varones el desarrollo de características más de tipo agresivo e instrumental, mientras que acentúa en las mujeres las de tipo relacional y expresivo (Rocha, 2008; Rebollo, Ruiz y García, 2017).

En cuanto a la interacción de género y edad, los resultados del presente estudio sugieren que los hombres de mayor edad probablemente tienden a presentar puntuaciones más altas que los de menor edad en el mencionado estilo agresivo, mientras que en las mujeres se presenta el fenómeno contrario: puntuaciones más bajas en el grupo de mayor edad. Tales hallazgos son 


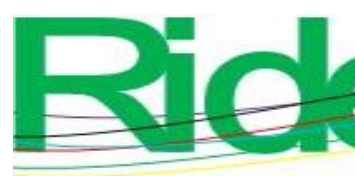

\section{Revista Iberoamericana para la Investigación y el Desarrollo Educativo \\ ISSN $2007-7467$}

congruentes con la llamada hipótesis de intensificación de género (Arnett, 2008; Steinberg, 2017). De acuerdo con dicha hipótesis, las diferencias de género en los individuos tienden a intensificarse, al menos temporalmente, durante la adolescencia debido a la presión social que pesa sobre los sujetos para adecuarse a los roles sociales establecidos. Según Arnett (2008), ello tiene por consecuencia, en el caso de los varones, la mayor presencia de conductas agresivas correspondientes con el estereotipo tradicional. Siguiendo todavía a este autor, en ese contexto "los adolescentes enfrentan la perspectiva intimidante de que los consideren hombres fracasados. Se defienden con agresiones verbales y aun físicas, cuando es necesario" (Arnett, 2008, p. 147).

En lo concerniente al cuarto objetivo del presente estudio, se planteó como hipótesis que se presentarían correlaciones positivas estadísticamente significativas: $a$ ) entre los estilos enfocado en sí mismo del Conflictalk y del CMMS; $b$ ) entre el estilo enfocado en la otra parte del CMMS y el enfocado hacia los otros (avestruz-pasivo), y $c$ ) entre los estilos enfocado en el problema del CMMS y del Conflictalk.

Como se pudo observar, las hipótesis primera y tercera se cumplieron ya que se presentaron las correlaciones señaladas de manera estadísticamente significativa. No obstante, no sucedió así en el caso del estilo enfocado en la otra parte (avestruz-evitativo), ya que este correlacionó significativamente solo con el estilo enfocado en sí mismo del cuestionario CMMS. A su vez, el estilo enfocado en la otra parte de este último cuestionario correlacionó de manera estadísticamente significativa con el estilo cooperativo del Conflictalk.

Una probable explicación de este último resultado puede obtenerse al considerar la naturaleza de los reactivos con que quedaron compuestas dichas escalas, tras sus correspondientes estudios de validación: en el caso del CMMS, los reactivos retenidos en la escala enfocado en la otra parte tienen un énfasis mayor en las conductas complacientes o de satisfacción de los deseos de la contraparte (Luna \& Laca, 2014), mientras que los correspondientes del Conflictalk presentan en su redacción un mayor énfasis hacia la evitación, tal como puede observarse en el Factor III de la tabla 2. Si bien el modelo de Kimsey y Fuller (2003) no contempla esta diferencia entre el estilo complaciente y el estilo evitativo, se trata de una distinción usual en la mayor parte de los modelos más reconocidos sobre estilos y estrategias de manejo de conflictos en la literatura sobre teoría de conflictos — por ejemplo, Blake y Mouton (1970), Galtung (2003), Kriesberg y Dayton (2012) y Rahim (2001), entre muchos otros. 


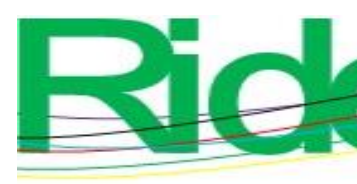

Revista Iberoamericana para la Investigación y el Desarrollo Educativo

ISSN $2007-7467$

En cuanto a las limitaciones del presente estudio y futuras líneas de trabajo derivadas del mismo, pueden señalarse al menos las siguientes. En primer lugar, como pudo observarse, es posible que existan otros estilos de manejo de conflictos empleados por los adolescentes en sus relaciones interpersonales, los cuales tal vez escapen al modelo de tres estilos que sirve de base al Conflictalk como, por ejemplo, el mencionado estilo complaciente del CMMS. Por tanto, se aconseja para futuros estudios analizar la relación del Conflictalk con otros instrumentos basados en modelos que contemplan un mayor número de estilos; entre ellos el modelo de Rahim (2001), cuyo cuestionario fue validado con adolescentes mexicanos en un estudio reciente (Luna, Valencia y Nava, 2018).

En segundo lugar, se aconseja contemplar muestras con un mayor rango de edad a fin de posibilitar un análisis más amplio del papel de la edad y el género, y con ello profundizar los hallazgos del presente estudio en cuanto a la interacción de estas variables.

En tercer lugar, se recomienda en futuros estudios examinar las posibles relaciones de las escalas del Conflictalk con variables asociadas a la dinámica de los conflictos interpersonales, tanto de carácter disposicional (personalidad, cognición, afectividad, bienestar psicológico, entre otras) como situacional (contexto relacional, por ejemplo), con el objetivo de lograr una mayor comprensión de las propiedades del instrumento.

Por último, sería recomendable realizar un nuevo estudio en el que la composición factorial obtenida en el presente trabajo se ponga a prueba a través de la técnica de análisis factorial confirmatorio (AFC), a fin de contar con datos que permitan evaluar las propiedades psicométricas del instrumento, pero desde la perspectiva confirmatoria.

\section{Conclusiones}

En conclusión, el presente estudio aporta datos relevantes para sustentar la validez y confiabilidad del Conflictalk y, en consecuencia, para considerarlo como un instrumento con propiedades psicométricas aceptables para la evaluación de los estilos de manejo de conflictos en muestras de adolescentes mexicanos de bachillerato. En particular, los resultados obtenidos apoyan la interpretación de que el instrumento posee una estructura trifactorial, con la cual es posible evaluar tres estilos de manejo de conflictos interpersonales: a) agresivo (rinoceronteagresivo), $b$ ) evitativo (avestruz-evitativo) y c) cooperativo (delfín-cooperativo). 


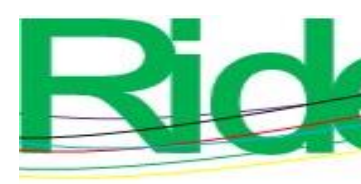

Revista Iberoamericana para la

Investigación y el Desarrollo Educativo

ISSN $2007-7467$

Además de lo anterior, el presente estudio aporta información relevante acerca de la capacidad del Conflictalk para identificar diferencias de género y edad que son congruentes con la literatura del área; en particular, en lo que corresponde al estilo agresivo, el cual mostró mayor presencia en adolescentes varones con respecto a mujeres, y en varones de mayor edad con respecto a los de menor edad.

Finalmente, el presente estudio también aporta datos a favor de la validez convergente y discriminante del Conflictalk al analizar la relación de sus tres escalas con las del instrumento CMMS.

No obstante lo anterior, para fortalecer y ampliar los resultados del presente estudio, se recomienda realizar nuevas investigaciones con muestras de rango de edad más amplio, así como estudios en los que se analice la estructura factorial del Conflictalk desde una perspectiva confirmatoria y su relación con más instrumentos que evalúen estilos de manejo de conflictos y otras variables de relevancia.

\section{Referencias}

American Psychological Association [APA]. (2017). Ethical principles of psychologists and code of conduct (2002, as amended 2010, 2016). United States: American Psychological Association. Retrieved from http://www.apa.org/ethics/code/ethics-code-2017.pdf.

Arnett, J. J. (2008). Adolescencia y adultez emergente. Un enfoque cultural (3. a ed.; trad. M. E. Ortiz). México: Pearson Educación.

Azzolini, A. B. (2017). Los derechos de la infancia. México: Universidad Nacional Autónoma de México y Editorial Porrúa.

Blake, R. R. and Mouton, J. S. (1970). The fifth achievement. Journal of Applied Behavioral Science, 6(4), 413-426.

Chang, L. C. and Zelihic, M. (2013). The study of conflict management among Taiwanese adolescents. Life Science Journal, 10(3), 1231-1241.

De Conti, M. (2014). The impact of competitive debate on managing the conflict communication strategies of Italian students. Argumentation and Advocacy, 51(2), 123-131.

De la Rosa, C. S., Ángeles, P. C. y Pérez, R. E. (2018). Tipo de mensaje ante el conflicto de estudiantes de colegio católico. Irene. Estudios de Paz y Conflictos, 1, 93-103. 
Donegani, C. G. and Séguin, D. G. (2018). Exploring affect and regulation as predictors of conflict in late childhood and early adolescence. Early Child Development and Care, 188(10), 1354-1367.

Filley, A. C. (1985). Solución de conflictos interpersonales (trad. C. Villegas García). México: Trillas.

Galtung, J. (2003). Paz por medios pacíficos. Paz y conflicto, desarrollo y civilización (trad. T. Toda). Bilbao, España: Bakeaz y Gernika Gogoratuz.

Garaigordobil, M. (2009). Evaluación del programa Dando pasos hacia la paz. Informe de investigación. Vitoria-Gasteiz, España: Servicio Central de Publicaciones del Gobierno Vasco. Recuperado de http://www.argia.eus/fitx/bestelakoak/bakerako-urratsak.pdf.

Garaigordobil, M. (2012). Cooperative conflict-solving during adolescence: relationships with cognitive-behavioural and predictor variables. Infancia y Aprendizaje, 35(2), 151-165.

Garaigordobil, M. (2017). Conducta antisocial: conexión con bullying/cyberbullying y estrategias de resolución de conflictos. Psychosocial Intervention, 26(1), 47-54. Recuperado de http://doi.org/10.1016/j.psi.2015.12.002.

Garaigordobil, M., Machimbarrena, J. M. y Maganto, C. (2016). Adaptación española de un instrumento para evaluar la resolución de conflictos (Conflictalk): datos psicométricos de fiabilidad y validez. Revista de Psicología Clínica con Niños y Adolescentes, 3(2), 59-67.

Garaigordobil, M. y Maganto, C. (2011). Empatía y resolución de conflictos durante la infancia y la adolescencia. Revista Latinoamericana de Psicología, 43(2), 255-266.

Garaigordobil, M., Maganto, C., Pérez, J. I. and Sansinenea, E. (2009). Gender differences in socioemotional factors during adolescence and effects of a violence prevention program. Journal of Adolescent Health, 44(5), 468-477.

Garaigordobil, M. and Martínez, V. (2015). The effectiveness of Cyberprogram 2.0 on conflict resolution strategies and self-esteem. Journal of Adolescent Health, 57(2), 229-234.

George, D. and Mallery, P. (2003). Spss for Windows step by step: A simple guide and reference. 11.0 Update (4. ${ }^{\mathrm{a}}$ ed.). Boston, United States: Allyn \& Bacon.

Hochhauser, M., Weiss, P. L. and Gal, E. (2018). Enhancing conflict negotiation strategies of adolescents with high functioning autism spectrum disorders through technology 


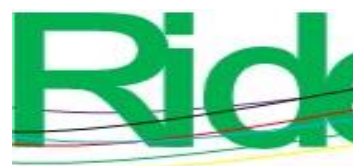

Revista Iberoamericana para la

Investigación y el Desarrollo Educativo

ISSN $2007-7467$

supported collaboration. Assistive Technology, 30(3), 107-118. Retrieved from https://doi.org/10.1080/10400435.2016.1268217.

Ibarra, E. y Jacobo, H. M. (2014). Adolescencia. Evolución del autoconcepto. Sinaloa, México: Universidad Autónoma de Sinaloa y Juan Pablos Editor.

IBM Corporation (2012). IBM SPSS Statistics for Windows (Version 21.0) [computer program]. Armonk, United States: IBM Corp.

Iglesias, J. L. (2013). Desarrollo del adolescente: aspectos físicos, psicológicos y sociales. Revista Pediatría Integral, 17(2), 88-93.

Kimsey, W. D. and Fuller, R. M. (2003). Conflictalk: an instrument for measuring youth and adolescent management message styles. Conflict Resolution Quarterly, 21(1), 69-78.

Kriesberg, L. and Dayton, B. W. (2012). Constructive conflicts: from escalation to resolution ( $4^{\text {th }}$ ed.). Lanham, United Kingdom: Rowman \& Littlefield Publishers.

Laca, F. A. (2005). Elección de estrategias de afrontamiento del conflicto bajo presión de tiempo. Bilbao, España: Editorial Universidad del País Vasco.

Laca, F. A., Alzate, R., Sánchez, M., Verdugo, J. and Guzmán, J. (2006). Communication and conflict in young mexican students: messages and attitudes. Conflict Resolution Quarterly, 14(1), 31-54.

Laca, F. A., Mejía, J. C. and Mayoral, E. G. (2011). Conflict communication, decision-making, and individualism in Mexican and Spanish university students. Psychology Journal, $8(1), 121-135$.

Lane, P. S., Ybarra, M., Zajac, J. D. and Vierra, T. (2005). Mediators and mentors: partners in conflict resolution and peace education. Journal of Peace Education, 2(2), 183-193.

Lloret, S., Ferreira, A., Hernández, A. y Tomás, I. (2014). El análisis factorial exploratorio de los ítems: una guía práctica, revisada y actualizada. Anales de Psicología, 30(3), 11511169.

Luna, A. C. A. (2014). Efecto de la comunicación parento-filial sobre los estilos personales de manejo de conflictos en adolescentes bachilleres. Uaricha, Revista de Psicología, 11(24), 118-133.

Luna, A. C. A. (2017). Relación entre estilos de manejo de conflictos y empatía multidimensional en adolescentes bachilleres. Revista Iberoamericana de las Ciencias Sociales y Humanísticas, 6(12), 80-106. 
Luna, A. C. A. (2018). Algunas contribuciones de la psicología del conflicto a la filosofía para la paz. Sincronía. Revista de Filosofía y Letras, 22(73), 3-24.

Luna, A. C. A., De Gante, A. y Gómez, M. A. (2018). Afrontamiento de conflictos en adolescentes de secundaria: su relación con violencia escolar, asertividad, autoestima y empatía. En Vallejo, R. y Ortega, M. C. (coords.), Distintos abordajes clínicos de las manifestaciones de la violencia (pp. 21-43). México: Distribuciones Fontamara.

Luna, A. C. A. y Laca, F. A. (2014). Estilos de mensajes en el manejo de conflictos en adolescentes y jóvenes mexicanos. Boletín de Psicología, 110, 37-51.

Luna, A. C. A. y Laca, F. A. (2017). Sexismo ambivalente y estilos de manejo de conflictos en estudiantes de bachillerato. Revista Iberoamericana para la Investigación y el Desarrollo Educativo, 8(15), 182-209. doi:10.23913/ride.v8i15.296

Luna, A. C. A., Mejía, J. C. y Laca, F. A. (2017). Conflictos entre pares en el aula y estilos de manejo de conflictos en estudiantes de bachillerato. Revista Evaluar, 17(1), 50-64.

Luna, A. C. A., Valencia, A. C. y Nava, J. M. (2018). Propiedades psicométricas del Inventario de Rahim en una muestra de adolescentes estudiantes de bachillerato. Revista Evaluar, $18(2), 75-90$.

Luna, A. C. A., Valencia, A. C., Nava, J. M. y Ureña, J. H. (2019). Avances en la línea de investigación sobre estilos de manejo de conflictos en adolescentes.RevistaEduc@rnos, $8(32), 155-180$.

Mejía, J. C. y Laca, F. A. (2006). Estilos de comunicación en el conflicto y confianza en las propias decisiones. Enseñanza e Investigación en Psicología, 11(2), 347-358.

Moral de la Rubia, J. (2016). Análisis factorial y su aplicación al desarrollo de escalas. En Landero, R. y González, M. T. (eds.), Estadística con SPSS y metodología de la investigación (pp. 387-443). México: Trillas.

Organización Mundial de la Salud [OMS]. (1986). La salud de los jóvenes: un desafío para la sociedad. Informe de un grupo de estudio de la OMS acerca de los jóvenes y la salud para todos en el año 2000 (trad. Organización Panamericana de la Salud). Ginebra, Suiza: Organización Mundial de la Salud.

Paris, S. (2009). Filosofía de los conflictos. Una teoría para su transformación pacífica. Barcelona, España: Icaria editorial. 
Pegalajar, M. C. (2018). Análisis del estilo de gestión del conflicto interpersonal en estudiantes universitarios. Revista Iberoamericana de Educación, 77(2), 9-30.

Rahim, M. A. (1983). A measure of styles of handling interpersonal conflict. Academy of Management Journal, 26(2), 368-376.

Rahim, M. A. (2001). Managing conflict in organizations ( $3^{\text {rd }}$ ed.). Westport, United States: Quorum Books.

Ramón, M. Á., García , M. P. y Olalde, A. J. (2019). Algunas consideraciones sobre la resolución de los conflictos escolares. Revista Conrado, 15(67), 135-142.

Rebollo, A., Ruiz, E. y García, R. (2017). Preferencias relacionales en la adolescencia según el sexo. Revista Electrónica de Investigación Educativa, 19(1), 58-72.

Rocha, T. E. (2008). La adolescencia: periodo crítico en la construcción del sexo. En Andrade, P., Cañas, J. L. y Betancourt, D. (comps.), Investigaciones psicosociales en adolescentes (pp. 15-44). Tuxtla Gutiérrez, México: Universidad de Ciencias y Artes de Chiapas y Universidad Nacional Autónoma de México.

Ross, R. and DeWine, S. (1988). Assessing the Ross-DeWine Conflict Management Message Style (CMMS). Management Communication Quarterly, 1, 389-413.

Santamaría-Cárdaba, N. (2019). ¿Cuál es el estatus de la educación para la paz en el ámbito científico actual? MODULEMA. Revista Científica sobre Diversidad Cultural, 3, 63-77.

Sociedad Mexicana de Psicología. (2010). Código ético del psicólogo (5. ${ }^{\mathrm{a}}$ ed.). México: Editorial Trillas.

Steinberg, L. (2017). Adolescence (11 $1^{\text {th }}$ ed.). New York, United States: McGraw-Hill Education.

Thomas, K. and Kilmann, R. (1974). Thomas-Kilmann Conflict Mode Instrument. Palo Alto, United States: Consulting Psychologists Press, Inc.

\section{Alejandro César Antonio Luna Bernal}

Doctor en Psicología por la Universidad de Colima. Actualmente se desempeña como profesor de tiempo completo en el Departamento de Filosofía de la Universidad de Guadalajara. Es miembro del Sistema Nacional de Investigadores (SNI), Nivel I, y forma parte del cuerpo académico "Adolescentes: mundo y vida" (UDG-CA-967). 